16 a 18 de outubro de 2019 - Campinas | Brasil

\title{
Impacto dos sexos sobre a relação entre dissecção de aorta e características estruturais e funcionais cardíacas.
}

\section{Matheus Fiori R. A. de Oliveira, Walter E. M. Rocha, Wilson Nadruz Junior.}

\section{Resumo}

A dissecção da aorta é uma emergência médica caracterizada pelo desenvolvimento agudo de uma delaminação na túnica íntima, permitindo uma falsa luz e fluxo sanguíneo na túnica média. Vários estudos têm sugerido que a presença de dissecção de aorta se associa com alterações na estrutura cardíaca, particularmente, com a hipertrofia ventricular esquerda (HVE). Estes dados indicam que as alterações aórticas e cardíacas possam compartilhar mecanismos fisiopatológicos comuns. Há ainda dados sugerindo que as relações entre o remodelamento vascular e cardíaco em homens e mulheres podem não ser similares. Contudo, ainda não se sabe se há diferenças de acordo com o sexo no remodelamento cardíaco em pacientes portadores de dissecção da aorta. Esse estudo compara as características morfológicas e funcionais cardíacas, avaliadas por ecocardiografia, entre homens e mulheres com doença da aorta.

\section{Palavras-chave: Dissecção de aorta, Hipertensão arterial, Hipertrofia cardíaca}

\section{Introdução}

A delaminação da camada intima arterial está diretamente relacionada com a hipertensão arterial, uma vez que o aumento da pressão sistêmica incide uma maior pressão na parede do vaso ${ }^{1}$. Modificações anatômicas e funcionais do sistema cardiovascular podem levar a uma resposta de Hipertrofia Ventricular Esquerda (HVE) ${ }^{2}$.

Alterações na estrutura cardíaca são marcadores independentes de pior prognostico cardiovascular e correspondem a um aumento da Massa Ventricular Esquerda (MVE) - Homens $>115 \mathrm{~g} / \mathrm{m}^{2}$ e mulheres $>95$ $\mathrm{g} / \mathrm{m}^{2}$ - e aumento da Espessura Relativa do Ventrículo Esquerdo (ERVE) - maior que 0,42 ${ }^{3}$. Estudos prévios indicam que o remodelamento cardíaco e aórtico podem compartilhar mecanisos comuns e mostram que há variações nos determinantes de alterações cardíacas e aórticas por sexo. Neste contexto, o objetivo desse estudo foi comparar as características morfológicas e funcionais cardíacas entre homens e mulheres com dissecção de aorta.

\section{Resultados e Discussão}

Tabela 1. Comparação entre homens e mulheres segundo critérios estudados

\begin{tabular}{lccc}
\hline \multicolumn{1}{c}{ Varíaveis } & Homens & Mulheres & $\boldsymbol{p}$ \\
\hline $\mathrm{n}(\%)$ & $215(70 \%)$ & $90(30 \%)$ & $\ldots$ \\
Idade & $56.7 \pm 12.0$ & $55.5 \pm 12.0$ & 0.44 \\
Stanford A & $129(60.0)$ & $60(66.7)$ & 0.27 \\
Índice de massa corpórea $\left(\mathrm{Kg} / \mathrm{m}^{2}\right)$ & $26.8 \pm 4.7$ & $27.1 \pm 6.1$ & 0.60 \\
Tabagismo & $98(46 \%)$ & $32(36 \%)$ & 0.1 \\
Diabetes mellitus & $16(7 \%)$ & $9(10 \%)$ & 0.45 \\
Doença arterial coronariana & $22(10 \%)$ & $14(15 \%)$ & 0.34 \\
Hipertensão arterial sistêmica & $166(77 \%)$ & $79(88 \%)$ & 0.034 \\
Pressão arterial sistólica $(\mathrm{mmHg})$ & $148.6 \pm 39.9$ & $151.3 \pm 40.1$ & 0.59 \\
Pressão arterial diastólica $(\mathrm{mmHg})$ & $87.1 \pm 25.0$ & $86.1 \pm 23.5$ & 0.74 \\
Creatinina (mg/dl) & $1.2[0.6]$ & $0.96[0.55]$ & $<0.001$ \\
\hline
\end{tabular}

Tabela 2. Características ecocardiográficas de indivíduos com dissecção de aorta

\begin{tabular}{lrll}
\hline \multicolumn{1}{c}{ Variáveis } & Homens & Mulheres & \multicolumn{1}{c}{$\boldsymbol{p}$} \\
\hline Aorta $(\mathrm{mm})$ & $41.8 \pm 8.4$ & $37.1 \pm 8.03<0.001$ \\
Diâmetro sistólico do ventrículo esquerdo $(\mathrm{mm})$ & $34.7 \pm 7.6$ & $31.3 \pm 6.2$ & $<0.001$ \\
Diâmetro diastólico do ventrículo esquerdo $(\mathrm{mm})$ & $53.8 \pm 8.0$ & $49.6 \pm 7.2$ & $<0.001$ \\
Espessura parede posterior ventrículo esquerdo $(\mathrm{mm})$ & $11.7 \pm 2.3$ & $11.0 \pm 2.2$ & 0.020 \\
Septo interventricular $(\mathrm{mm})$ & $12.0 \pm 2.6$ & $11.3 \pm 2.4$ & 0.030 \\
Índice de massa ventrículo esquerdo $\left(\mathrm{g} / \mathrm{m}^{2}\right)$ & $149.5 \pm 61$ & $136.4 \pm 49$ & 0.07 \\
Espessura relativa ventrículo esquerdo & $0.44 \pm 0.11$ & $0.45 \pm 0.12$ & 0.45 \\
Fração de ejeção $(\%)$ & $64.2 \pm 10.8$ & $65.9 \pm 8.4$ & 0.19
\end{tabular}

Figura 1 - Remodelamento cardíaco entre homens e mulheres com dissecção de aorta.

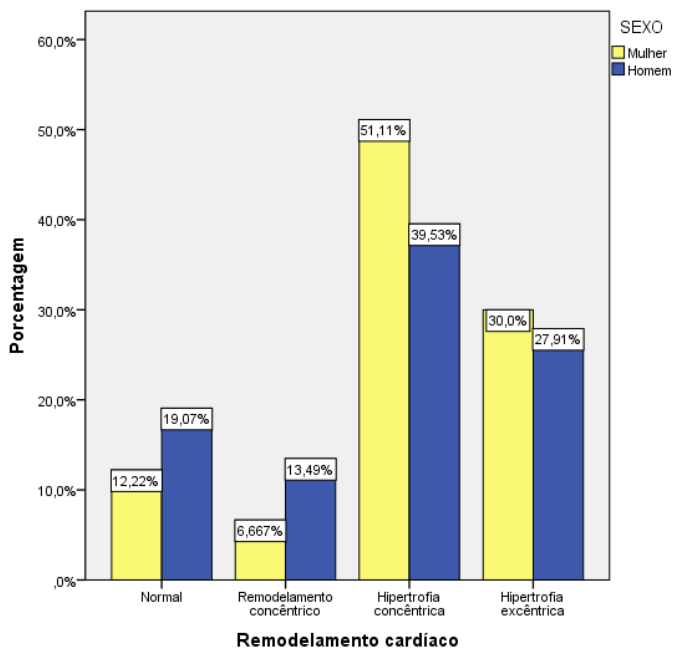

Foram analisados 300 indivíduos, sendo 215 (70\%) do sexo masculino e 90 (30\%) do sexo feminino. Não houve diferença estatística em relação à média de idade, IMC, tipo de dissecção, tabagismo, diabetes mellitus, doença arterial coronariana, pressão sistólica e diastólica, MVE, ERVE, fração de ejeção (Tabelas 1 e 2). Contudo, houve tendência a maior prevalência de hipertrofia concêntrica em mulheres $(p=0,06)$ (Figura 1).

\section{Conclusões}

Nessa população estudada, a hipertrofia concêntrica predomina entre as alterações cardíacas nos pacientes com dissecção de aorta, e, apesar de não ser diferente estatisticamente, mostrou tendência à maior prevalência no sexo feminino.

\section{PIBIC, FCM/Unicamp}

\section{Agradecimentos}

\footnotetext{
1 - Hagan PG, Nienaber CA, Isselbacher EM, et al. The International Registry of Acute Aortic Dissection (IRAD). JAMA. 2000;283(7):897. doi:10.1001/jama.283.7.897

2 - Wollert KC, Drexler H. Regulation of cardiac remodeling by nitric oxide: Focus on cardiac myocyte hypertrophy and apoptosis. Heart Fail Rev. 2002;7(4):317-325 doi:10.1023/A:1020706316429

3 - Nadruz W. Myocardial remodeling in hypertension. J Hum Hypertens. 2015;29(1):1-6. doi:10.1038/jhh.2014.36
} 